\title{
Investimento parental e desenvolvimento da criança
}

\author{
Eulina Rocha Lordelo \\ Carine Bastos da França \\ Lígia Maria dos Santos Lopes \\ Maria del Pilar Ogando Dacal \\ Cláudio Seal Carvalho \\ Raquel Cardoso Guirra \\ Anderson Almeida Chalub \\ Universidade Federal da Bahia
}

\begin{abstract}
Resumo
Segundo a teoria do investimento parental, seria esperada uma relação entre condições de criação da mãe e sua carreira reprodutiva e, por conseqüência, seus padrões de cuidado aos filhos, com repercussões no desenvolvimento das crianças. Esta pesquisa buscou verificar essas relações, em amostra de 37 mães e seus filhos entre um e quatro anos, de um bairro pobre de Salvador, Bahia. Foi investigada a história familiar e reprodutiva das mães, associada a resultados desenvolvimentais das crianças, medidos através das escalas Bayley e WIPPSI$\mathrm{R}$, em quatro avaliações realizadas ao longo de três anos. Foram encontradas correlações entre condições de criação da mãe e sua carreira reprodutiva subseqüente. Por sua vez, esses padrões mostraram-se modestamente relacionados ao desenvolvimento cognitivo de seus filhos, favorecendo as crianças cujas mães iniciaram sua vida reprodutiva mais tarde. Os resultados são, em geral, compatíveis com a teoria do investimento parental. Limitações do estudo e perspectivas futuras são discutidas.
\end{abstract}

Palavras-chave: investimento parental; desenvolvimento cognitivo; estratégias reprodutivas; psicologia evolucionista

\begin{abstract}
Parental investment and child development. According to the parental investment theory, it would be expected an association between maternal family environment and her reproductive behavior and, as a consequence, her patterns of parental investment on her children, with effects on their development. This study aimed to verify that association, in a sample of 37 mothers and their children (one to four years old), in a poor neighborhood of Salvador, in the state of Bahia, Brazil. We studied the family and reproductive history of the mothers and assessed the cognitive development of children, through Bayley and WIPPSI-R scales, in four assessments during three years. We found correlation between the raising environment of the mother and her subsequent reproductive patterns. These patterns were related to cognitive development of children, favoring children whose mothers started their reproductive life later. The results are, in general, compatible with the parental investment theory. Limitations of the study and future perspectives are discussed.
\end{abstract}

Keywords: parental investment, cognitive development, reproductive strategies, evolutionary psychology

$\mathrm{N}$ a teoria do investimento parental, o ciclo vital dos indivíduos como um todo está ligado aos seus interesses reprodutivos e as condições ecológicas em que eles vivem afetam suas estratégias, concebidas na dimensão quantidade/qualidade. Em princípio, a espécie humana caracteriza-se pelo alto investimento parental, entretanto, diferenças entre os indivíduos permitem caracterizar dois padrões prototípicos de trajetórias desenvolvimentais: o primeiro estilo (Tipo 1) caracteriza o indivíduo que, na infância, experimenta um ambiente de escassez de recursos, com baixo investimento parental e clima familiar insatisfatório; o segundo (Tipo 2) descreve o indivíduo que, vivendo uma situação de abundância de recursos, experimenta um alto investimento parental e um clima familiar positivo. Além de conseqüências psicológicas nas relações de apego entre criança e pais, os dois tipos de experiências levariam a diferenças no início da puberdade, com o Tipo 1 amadurecendo mais cedo que o Tipo 2. Essas trajetórias continuariam a se diferenciar nos estilos reprodutivos subseqüentes, sendo o Tipo 1 mais quantitativo, significando isso mais filhos, menor espaçamento 
entre os nascimentos e baixo investimento parental; ao contrário, o protótipo do Tipo 2 seria mais qualitativo, implicando menos filhos, maior espaçamento entre os filhos e alto investimento parental (Keller, 1996).

O investimento parental é definido geralmente pela maior ou menor quantidade de cuidado biológico e/ou psicológico dispensado à prole. As decisões de investimento parental são baseadas nas condições ecológicas que os pais vivenciam, incluindo a ordem de nascimento da criança, as quais se expressam na prevalência de certos estilos de cuidado. Essas estratégias são, obviamente, inconscientes (Keller, 1996).

Segundo Keller (1998a), a primeira fase do desenvolvimento humano pode ser caracterizada pela tarefa de aquisição de uma matriz social primária, adaptativa em relação ao ambiente no qual o indivíduo se desenvolve. Essa matriz social, construída nas interações com os cuidadores, capacitaria o indivíduo a adotar estratégias reprodutivas em consonância com os desafios do ambiente circundante. Desse modo, supõe-se que os sistemas de cuidado a que o indivíduo é exposto durante os primeiros anos de vida têm conseqüências importantes nos resultados desenvolvimentais, as quais, por sua vez, poderão afetar a próxima geração. A teoria supõe que os estilos reprodutivos dos indivíduos são principalmente não-intencionais, não conscientes e implícitos, sendo regulados por uma psicologia complexa, capaz de processar e integrar informação, motivações, desejos, crenças, atitudes e comportamentos referentes ao sucesso reprodutivo (Keller, 1996, 1998a).

Essa teoria tem o potencial de integrar dimensões biológicas e culturais a uma psicologia do desenvolvimento humano. A cadeia de raciocínio que leva a problemas de pesquisa é: existe ampla variabilidade quanto aos processos de desenvolvimento e seus resultados, o que deve ser explicado por uma teoria que não viole a biologia evolucionária. O modo como o indivíduo é criado influencia as adaptações do organismo adulto. Então, quais os determinantes dos modos de criação? Conquanto cultura e variáveis pessoais como personalidade sejam vistas como determinantes, elas não podem ser arbitrárias e devem estar relacionadas com estratégias de adaptação dos organismos, que por sua vez estão relacionadas com condições ecológicas específicas. Então, como cultura e biologia se ligam para influenciar o desenvolvimento?

Entre as diversas linhas de pesquisa relacionadas a essa pergunta geral, destaca-se o trabalho que vem sendo realizado por Belsky e colaboradores (Belsky, Steinberg, \& Draper, 1991; Moffit, Caspi, Belsky, \& Silva, 1992). Eles estudaram uma amostra de 326 meninas adolescentes dos Estados Unidos, criadas em diferentes condições. Foram encontradas diferenças significativas no início da puberdade e idade da menarca, mais cedo entre aquelas meninas criadas sem os pais, bem como entre as que tiveram experiência de ambiente familiar conflituoso. Entretanto, não foi possível excluir hipóteses alternativas, dada a ausência de controle sobre variações biológicas condicionadas geneticamente. Mas um es- tudo realizado mais tarde por Graber, Brooks-Gunn e Warren (1995), controlando a idade da menarca das mães, encontrou a mesma relação.

Pettit e Bates (1999), seguindo a mesma linha, mas enfatizando a qualidade do investimento parental paterno, estudaram 173 meninas nos Estados Unidos e encontraram uma associação positiva entre presença do pai nos primeiros anos de vida, especialmente quando o envolvimento do pai era alto, e menarca mais tardia.

Outros estudos que se seguiram têm investigado o problema através de diversos modelos alternativos. Por exemplo, Ellis e Garber (2000), num estudo com 87 adolescentes, testaram a hipótese de que uma história de problemas psicopatológicos da mãe, mediada por relações familiares conflituosas e ausência do pai/presença de um padrasto, produziria uma puberdade mais precoce nas filhas, o que foi confirmado pelos resultados encontrados, tendo também sido possível separar os efeitos do clima familiar estressante daqueles atribuídos à ausência do pai e presença de padrasto, ambos importantes preditores da idade da menarca.

Por outro lado, Barber (2003) investigou a hipótese de que o número de nascimentos de genitores solteiros aumentaria seguindo a diminuição de expectativas de investimento parental, medida por altas taxas de desemprego de homens adultos, baixa renda, desproporção entre o número de homens e mulheres, e alta taxa de gravidez na adolescência. O estudo utilizou dados secundários de estatísticas sóciodemográficas de 85 países com desenvolvimento sócio-econômico acima da média mundial. Os resultados mostraram que a taxa de nascimentos de mães solteiras declina seguindo o aumento de expectativas de investimento paternal e aumenta conforme a elevação de escolaridade da mulher, essas duas tendências como explicações prováveis dos determinantes da gravidez fora do casamento.

Quinlan, Quinlan e Flinn (2003) estudaram o tempo de desmame em uma pequena comunidade caribenha, buscando testar as predições da teoria do investimento parental. Eles coletaram dados de 101 crianças e encontraram uma associação negativa entre ausência do pai e desmame precoce. Entretanto, as demais hipóteses decorrentes da teoria foram rejeitadas, especificamente as que propunham relação entre o tempo de amamentação e a disponibilidade de parentes mulheres, renda familiar, número de crianças dependentes e idade da mãe ao nascimento. O estudo propôs que a teoria do investimento parental deve incluir no seu modelo as variáveis relativas ao custo de oportunidades para cuidado prolongado, as demandas para o trabalho feminino e a importância do investimento em "capital corporificado". Eles concluem que o modelo original de Trivers-Willard pode não ser suficiente para explicar as estratégias reprodutivas humanas, uma vez que as relações entre os componentes do investimento parental são complexas e dependentes do contexto, envolvendo, provavelmente, mais elementos, com destaque para o tipo de trabalho da mulher e a composição familiar.

Ellis et al. (2003) investigaram longitudinalmente duas amostras de meninas dos Estados Unidos e Nova Zelândia, 
entre cinco e 18 anos de idade, controlando variáveis correlatas associadas, como problemas de externalização de comportamento e contexto sócio-econômico e encontraram uma clara associação entre ausência do pai na infância e risco de atividade sexual, bem como gravidez precoce na adolescência, sendo a relação ainda mais forte quando a ausência do pai ocorre desde os primeiros anos de vida. O estudo discute especificamente a adequação da explicação que atribui a gravidez na adolescência a estressores sócio-econômicos, tomando a ausência do pai como um desses estressores. Com base nos dados, os autores concluem que esse modelo explicativo é inadequado e destacam o papel crucial que a ausência do pai desempenha no ciclo de desenvolvimento das crianças.

No Brasil, a maioria dos estudos realizados sobre idade da menarca tem encontrado uma tendência à antecipação da idade, conforme a melhora de condições de vida da população (Picanço, 1995; Tavares et al., 2000; Zerwes \& Simões, 1993). Um estudo de Lago (2002), entretanto, que controlou o nível de escolaridade dos pais, encontrou que, embora a antecipação da idade da menarca seja uma tendência secular geral na população, ela é mais acentuada entre as meninas cujos pais têm nível de escolaridade mais baixo.

Também Schor (1990), estudando uma amostra de 2.588 mulheres da região de Santo André (Grande São Paulo), encontrou correlações positivas entre idades da menarca, primeira relação sexual e primeira gravidez.

Devido à evidência de que a desnutrição tende a retardar a puberdade (Hrdy, 2000), é importante controlar as variáveis envolvidas, de modo a identificar os fatores relevantes nas estratégias reprodutivas empregadas e seus efeitos no desenvolvimento das crianças. Por outro lado, padrões reprodutivos e desenvolvimento da criança são reconhecidamente sensíveis às condições sócio-econômicas e culturais vigentes, razão pela qual há a necessidade de estudar amostras de diferentes contextos ecológicos, de modo a estabelecer a generalidade do fenômeno em estudo. Assim, a presente pesquisa foi planejada numa população brasileira do mesmo nível sócio-econômico, residente no mesmo bairro, num esforço de reduzir a influência de variáveis extrínsecas ao problema. Desse modo, o objetivo do presente estudo foi relacionar condições de criação da mãe, estilos reprodutivos e desenvolvimento da criança.

\section{Método}

\section{Contexto do estudo e participantes}

Este trabalho constitui um segmento de um estudo maior, intitulado "Creche como sistema ecológico de desenvolvimento”, consistindo em uma investigação descritiva longitudinal, sobre os constituintes do ecossistema desenvolvimental - crenças e práticas de cuidado, condições ambientais relevantes e seus potenciais impactos no desenvolvimento da criança. O presente conjunto de dados aborda a história familiar e reprodutiva das mães como um desses elementos e sua associação ao desenvolvimento da criança, medido através da dimensão desenvolvimento cognitivo.

Participaram do estudo 37 crianças (um a três anos de idade no começo do estudo) e suas mães, vivendo num bairro pobre da periferia de Salvador. Durante os três anos do estudo, cinco casos foram perdidos, reduzindo a amostra para 32 crianças passíveis de comparação em todas as etapas da avaliação. As perdas foram causadas por mudanças de bairro das famílias e impossibilidade de localizar os novos endereços. As primeiras famílias participantes do estudo foram contatadas através da creche freqüentada pelas crianças. Essas pessoas que aceitaram participar da pesquisa indicaram novas famílias com condições semelhantes e moradoras do mesmo bairro. O projeto de pesquisa foi apresentado e discutido com os participantes, bem como seus direitos e contribuição esperada. Todos os procedimentos para garantir o respeito aos participantes foram seguidos, inclusive a assinatura de Termo de Consentimento.

O bairro onde o estudo foi realizado está situado numa área pobre de Salvador, no qual atuam diversas ONGs nas áreas de educação e saúde. Descrições detalhadas das condições sócio-econômicas da área estão disponíveis em AVSI (1999), Moreira (1999) e Moreira e Lordelo (2002).

\section{Instrumentos}

Entrevista com a mãe: informações demográficas, história da família e carreira reprodutiva

Foram conduzidos procedimentos de adaptação de um roteiro de entrevista, proposto por Keller (1998b), focalizando a história da família e carreira reprodutiva da mãe, bem como informações demográficas.

\section{Escalas Bayley e WIPPSI-R}

A Escala Bayley para o Desenvolvimento Infantil (Bayley, 1993) é constituída de três sub-escalas (Mental, Motora e Comportamental), avaliando o desempenho da criança em diversas tarefas propostas nessas áreas, com resultados expressos em escores normalizados (média 100 e desvio padrão 15). O presente estudo utilizou os escores obtidos na escala mental. Devido à falta de validação da escala à população brasileira, foi ignorada a escala classificatória, sendo utilizados os escores individuais normalizados para comparação entre os grupos. A escala vem sendo usada em vários estudos brasileiros, mostrando-se capaz de captar mudanças desenvolvimentais e diferenciar grupos (Eickmann, Lira, \& Lima, 2002; Gagliardo, Gonçalves, Lima, Francozo, \& Aranha Neto, 2004; Mello, Gonçalves, \& Souza, 2004).

A escala WIPPSI-R (Wechsler Preschool Scale of Intelligence - Revised) é um conjunto de 12 sub-testes utilizados na avaliação da inteligência de crianças de três anos a sete anos e três meses de idade, envolvendo diversas capacidades perceptuais, motoras e verbais. De acordo com o autor (Wechsler, 1989), os itens de teste foram escolhidos com base em uma concepção de inteligência como um constructo global e multidimensional. O desempenho da criança em cada tarefa/item é totalizado num escore bruto que, por sua vez, é convertido para uma escala normalizada (média 100 e desvio 
padrão 15). Para a análise dos dados, também foram utilizados os escores normalizados obtidos pela criança, ignorando-se as classificações indicadas pelos escores, pelas mesmas razões já mencionadas quanto à Escala Bayley.

\section{Procedimentos de coleta e análise dos dados}

As entrevistas com as mães, bem como a maioria das avaliações das crianças foram realizadas numa sala da creche. Em alguns casos, as escalas foram aplicadas nas casas das crianças, quando houve dificuldades para a mãe trazer seus filhos, ou quando as crianças relutavam em responder às tarefas solicitadas, por um provável efeito de estranhamento do ambiente. A aplicação dos testes completos foi realizada geralmente em uma única sessão, mas em alguns casos, as tarefas foram espaçadas em vários dias, até que se pudesse ter certeza de que o melhor desempenho possível da criança tinha sido obtido. Em geral, uma avaliação completa durava 50 minutos, em média.

Freqüências e médias foram calculadas e os grupos arranjados conforme as variáveis examinadas: arranjo de criação da mãe, idades dos eventos relevantes da vida reprodutiva e ordem de nascimento da criança. Testes de correlação nãoparamétrica (Spearman) foram aplicados às variáveis contínuas e a técnica de análise de variância foi utilizada para comparar o desenvolvimento das crianças conforme os grupos formados.

\section{Resultados}

No início do estudo, as crianças tinham em média 21,9 meses, como pode ser visto na Tabela 1 . Meninos e meninas estiveram bem balanceados na amostra e a renda familiar média esteve um pouco acima $(\mathrm{R} \$ 257,60)$ do salário mínimo vigente na época. A idade média das mães foi 27,1 e a escolaridade média chegou a 6,1 anos de escola. No conjunto, a amostra é relativamente homogênea, como pode ser visto nas medidas de desvio padrão, com a exceção da renda familiar.

De acordo com a teoria do investimento parental, seria esperada uma relação entre condições de criação das mães e sua carreira reprodutiva e, por conseqüência, seus padrões
Tabela 1

Caracterização da população no início do estudo

\begin{tabular}{lcc}
\hline & $\begin{array}{c}\text { Freqüência } \\
\text { e médias }\end{array}$ & $\begin{array}{c}\text { Desvio } \\
\text { padrão }\end{array}$ \\
\hline Meninas & $56,0 \%$ & - \\
Idade da criança (meses) & 21,9 & 7,0 \\
Renda familiar (R\$) & 257,60 & 232,6 \\
Idade da mãe (anos) & 27,1 & 6,1 \\
Escolaridade da mãe (anos) & 6,1 & 3,0 \\
Número de casos & 37 & - \\
\hline
\end{tabular}

${ }^{(*)}$ No começo do estudo o salário mínimo nacional era de R\$ 240,00

de cuidado aos filhos, com repercussões no desenvolvimento das crianças.

Quanto à primeira relação, as mães da amostra foram agrupadas conforme dois arranjos de criação. Como pode ser visto na Tabela 2, as mães que foram criadas com os dois genitores diferenciam-se daquelas que foram criadas em outros arranjos (apenas a mãe, apenas o pai, parentes ou outras pessoas) quanto ao número de casamentos, idade do primeiro casamento e idade do nascimento do primeiro filho, sendo essas diferenças estatisticamente significativas. Nesses três indicadores, as diferenças existentes apontam para o padrão de investimento parental mais quantitativo entre as mulheres criadas apenas com a mãe, ou o pai, outros parentes ou não parentes, em termos de idade mais baixa do início da vida reprodutiva e maior número de parceiros, em comparação com as mulheres que foram criadas com os dois progenitores. Uma diferença de sete décimos (correspondendo a aproximadamente sete meses) foi encontrada na idade da menarca entre os dois grupos, mais cedo entre as mulheres que não foram criadas pelos dois pais. Apesar de não-significativa estatisticamente, essa diferença pode ser mencionada, pelo menos como uma possibilidade a ser mais investigada em estudos com amostras maiores. A mesma tendência aparece também no indicador número de filhos, com a média de 2,0 filhos para as mulheres criadas com o pai e a mãe e 2,9 nos demais arranjos, diferença estatisticamente não-significativa, entretanto.

Tabela 2

Médias de indicadores da história reprodutiva da mãe conforme suas condições de criação

\begin{tabular}{|c|c|c|c|c|c|c|}
\hline & \multicolumn{2}{|c|}{ Infância com pai e mãe } & \multicolumn{2}{|c|}{ Outros arranjos } & \multicolumn{2}{|c|}{ ANOVA } \\
\hline & Média & $D P$ & Média & $D P$ & $F$ & $p$ \\
\hline Idade da menarca & 13,3 & 1,7 & 12,6 & 2,6 & 1,12 & ns \\
\hline Idade no primeiro casamento & 22,4 & 6,1 & 18,2 & 4,0 & 4,66 & $*$ \\
\hline Número de casamentos & 0,9 & 0,5 & 1,5 & 1,0 & 4,96 & $*$ \\
\hline Idade no nascimento do $1^{\circ}$ filho & 22,6 & 5,5 & 19,2 & 2,9 & 4,78 & $*$ \\
\hline Número de filhos & 2,0 & 1,2 & 2,9 & 2,3 & 2,05 & ns \\
\hline Número de casos & \multicolumn{2}{|c|}{22} & \multicolumn{2}{|c|}{15} & - & - \\
\hline
\end{tabular}

$* p \leq 0,05 ; n s=$ não-significativo 
A idade da menarca mostrou-se associada com dois indicadores de padrão de história reprodutiva, as idades do primeiro casamento e do nascimento do primeiro filho. Conforme pode ser visto na Tabela 3, foram encontradas correlações positivas entre a idade da primeira menstruação e idade do primeiro casamento $\left(r_{s}=0,41 ; p \leq 0,05\right)$ e idade do primeiro filho $\left(r_{s}=0,36 ; p \leq 0,05\right)$. Por sua vez, a idade da primeira união conjugal está positivamente correlacionada com a idade no nascimento do primeiro filho $\left(r_{s}=0,81 ; p \leq 0,01\right)$.

Assim, há base para sustentar algumas hipóteses decorrentes da teoria do investimento parental, quanto à conexão entre calendário maturacional e alguns aspectos da carreira reprodutiva da mulher. $\mathrm{O}$ elo seguinte, a conexão entre carreira reprodutiva e resultados desenvolvimentais, também recebe algum apoio dos dados, como pode ser visto na Tabela 4. Em todas as avaliações, observa-se uma tendência a aumento do Índice de Desenvolvimento Mental (IDM) conforme aumenta a idade do primeiro casamento da mulher, sendo essas diferenças estatisticamente significativas em três das quatro avaliações. Especialmente na terceira avaliação, quando as crianças tinham uma média de 39,8 meses de idade, a média do IDM para crianças cujas mães se casaram com idade menor ou igual a 15 anos foi de 83,4, subindo para 89,9 no grupo com mães casadas entre e 16 e 21 anos e, finalmente, atingindo 99,1 para o grupo de crianças cujas mães casaramse somente a partir de 22 anos $\left(F_{1,32}=5,60 ; p \leq 0,01\right)$.

A mesma tendência é observada quando se analisa a idade da mãe no nascimento do primeiro filho, como seria de se esperar no contexto sócio-demográfico brasileiro, uma vez que a gravidez é um poderoso determinante do casamento informal nos ambientes urbanos brasileiros. A idade do primeiro casamento está altamente correlacionada com a idade da mulher no nascimento do primeiro filho. Assim, observase também um efeito dessa variável no desenvolvimento cognitivo da criança, como pode ser visto na Tabela 5. Em geral, os índices de desenvolvimento mental são mais altos quanto mais alta é a idade da mãe no nascimento do primeiro filho (as crianças deste estudo não são necessariamente os primeiros filhos das mulheres). Em duas das quatro avaliações, as diferenças são estatisticamente significativas; por exemplo, na terceira avaliação, as médias de desenvolvimento cognitivo variam de 82,0 para crianças cujas mães tiveram o primeiro filho até 16 anos, subindo para 90,8 para os filhos de mulheres que tiveram a primeira gravidez entre 17 e 19 anos e chegando a 97,8 no grupo cujas mães tiveram o primeiro filho a partir dos 20 anos.

Tabela 3

Correlações (Spearman) entre variáveis da carreira reprodutiva

\begin{tabular}{lcccc}
\hline & 1 & 2 & 3 & 4 \\
\hline 1. Idade da menarca & - & - & - & - \\
2. Idade da primeira união conjugal & $0,41 *$ & - & - & - \\
3. Idade no nascimento do primeiro filho & $0,36 *$ & $0,81 * *$ & - & - \\
4. Número de filhos & $-0,10$ & $-0,33$ & $-0,14$ & - \\
\hline
\end{tabular}

$* p \leq 0,05 \quad * * p \leq 0,01$

Tabela 4

Médias do Índice de Desenvolvimento Mental (IDM) por idade do primeiro casamento (formal ou informal)

\begin{tabular}{lccccccccc}
\hline & Idade (meses) & \multicolumn{2}{c}{ Até 15 anos } & 16 a 21 anos & 22 anos ou mais & ANOVA \\
\cline { 2 - 9 } & Média & Média & $\boldsymbol{D P}$ & Média & DP & Média & $\boldsymbol{D P}$ & $\boldsymbol{F}$ & $\boldsymbol{p}$ \\
Média do IDM 1 & 24,9 & 82,2 & 7,2 & 88,8 & 11,3 & 94,9 & 10,4 & 3,16 & $*$ \\
Média do IDM 2 & 31,6 & 83,2 & 11,6 & 88,7 & 12,4 & 95,2 & 9,0 & 2,83 & $\boldsymbol{n} \boldsymbol{5}$ \\
Média do IDM 3 & 39,8 & 83,4 & 7,7 & 89,9 & 14,3 & 99,1 & 6,0 & 5,60 & $* *$ \\
Média do IDM 4 & 50,8 & 92,8 & 10,9 & 86,8 & 10,5 & 100,5 & 12,0 & 4,80 & $* *$ \\
Número de casos & - & \multicolumn{2}{c}{5} & \multicolumn{2}{c}{11} & & 16 & - & - \\
\hline
\end{tabular}

$* p \leq 0,05 ; \quad * * p \leq 0,01 ; \quad n s=$ não-significativo 
Esse resultado não pode ser explicado pela juventude da mãe em si mesma, uma vez que não foi encontrada correlação entre a idade da mãe por ocasião do nascimento da criança e o desempenho da criança em desenvolvimento cognitivo.

Finalmente, observa-se uma tendência à diminuição das médias do IDM conforme a criança ocupa uma ordem de nascimento mais tardia. Embora essas diferenças não sejam estatisticamente significativas, elas são suficientemente regulares para, pelo menos, serem mencionadas como uma possibilidade, a ser explorada em outros estudos, uma vez que tendem a ocorrer em mais de uma avaliação. Como se vê na Tabela 6, em três das quatro avaliações, há um decréscimo suave e constante nas médias do IDM, conforme a criança seja o primeiro, o segundo, ou o terceiro (ou mais) filho da mulher.

\section{Discussão e conclusões}

Em geral, os resultados aqui apresentados apóiam a hipótese de que as condições de criação da mãe estão relacionadas ao seu calendário maturacional e este ao padrão de vida reprodutiva, em termos de idade, número de casamentos e número de filhos. Assim, os dados encontrados numa amostra brasileira estão de acordo com o obtido em alguns estudos realizados em outros países, como é o caso de Belsky et al. (1991) e de Moffit et al. (1992). A partir daí, aparecem associações entre a idade da primeira união conjugal e do primeiro filho com os resultados de desenvolvimento cognitivo da criança. Assim, condições ecológicas vigentes nos primeiros anos de vida, particularmente a presença de pai e mãe no ambiente de criação da mãe, parecem estar ligadas aos padrões reprodutivos da mulher e estes padrões, por sua vez, ligam-se a resultados no desenvolvimento das crianças.

A natureza dessa ligação, como previsto na teoria (Keller, 1996) envolve a mediação dos processos de apego, que se supõe seja afetado pelo grau de investimento parental, de forma que baixo investimento parental resulta em apego inseguro e este, por sua vez, afeta negativamente o desenvolvimento, em diversas esferas, inclusive o desenvolvimento cognitivo, como evidenciado em alguns estudos (Moss, Cyr, \& Dubois-Comtois, 2004; Moss \& St-Laurent, 2001; Stams, Juffer, \& van Ijzendoorn, 2002).

Tratando-se de uma hipótese que liga eventos de diferentes momentos no tempo, envolvendo três gerações, não é possível controlar, num único estudo empírico, as muitas variáveis relevantes, que poderão ter um papel nos fenômenos estudados. Por exemplo, as interações entre recursos materiais, ambiente familiar e contexto social, que podem afetar substancialmente o significado de uma variável como ambiente de criação, permanecem inexploradas. Também a conexão entre calendário reprodutivo e investimento parental requereria medidas específicas e controle de variáveis relevantes. No presente estudo, indicadores de investimento parental como

Tabela 5

Médias do Índice de Desenvolvimento Mental (IDM) por idade da mãe no nascimento do primeiro filho

\begin{tabular}{lcccccccccc}
\hline & Idade (meses) & \multicolumn{2}{c}{ Até 16 anos } & \multicolumn{2}{c}{17 a 19 anos } & 20 anos ou mais & \multicolumn{2}{c}{ ANOVA } \\
\cline { 2 - 10 } & Média & Média & $D P$ & Média & $D P$ & Média & DP & $F$ & $p$ \\
\hline Média do IDM 1 & 24,9 & 80,2 & 6,6 & 91,5 & 12,4 & 92,0 & 10,1 & 2,04 & $\boldsymbol{n}$ \\
Média do IDM 2 & 31,6 & 78,2 & 4,0 & 93,1 & 11,2 & 91,9 & 11,2 & 3,12 & $*$ \\
Média do IDM 3 & 39,8 & 82,0 & 8,1 & 90,8 & 13,1 & 97,8 & 8,7 & 4,13 & $*$ \\
Média do IDM 4 & 50,8 & 89,5 & 9,3 & 94,5 & 16,1 & 95,8 & 11,2 & 0,39 & ns \\
Número de casos & - & \multicolumn{2}{c}{4} & \multicolumn{2}{c}{11} & & \multicolumn{2}{c}{17} & - & - \\
\hline
\end{tabular}

$* p \leq 0,05 ; \quad n s=$ não-significativo

Tabela 6

Índice de Desenvolvimento Mental (IDM) por ordem de nascimento da criança

\begin{tabular}{|c|c|c|c|c|c|c|c|c|c|}
\hline & \multirow{2}{*}{$\begin{array}{c}\text { Idade (meses) } \\
\text { Média }\end{array}$} & \multicolumn{2}{|c|}{ Primeiro filho } & \multicolumn{2}{|c|}{ Segundo filho } & \multicolumn{2}{|c|}{ Terceiro ou mais } & \multicolumn{2}{|c|}{ ANOVA } \\
\hline & & Média & $D P$ & Média & $D P$ & Média & $D P$ & $F$ & $p$ \\
\hline Média do IDM 1 & 24,9 & 92,3 & 8,6 & 91,6 & 15,0 & 87,0 & 10,6 & 0,80 & $n s$ \\
\hline Média do IDM 2 & 31,6 & 93,0 & 12,0 & 89,8 & 10,7 & 88,5 & 11,7 & 0,52 & ns \\
\hline Média do IDM 3 & 39,8 & 93,7 & 11,2 & 99,2 & 8,1 & 87,3 & 12,7 & 2,82 & $n s$ \\
\hline Média do IDM 4 & 50,8 & 97,9 & 12,7 & 95,2 & 14,1 & 88,6 & 10,2 & 1,59 & $n s$ \\
\hline Número de casos & - & \multicolumn{2}{|c|}{15} & \multicolumn{2}{|c|}{8} & \multicolumn{2}{|c|}{9} & - & - \\
\hline
\end{tabular}

$n s=$ não-significativo 
tempo de amamentação e padrões de cuidado foram explorados ligeiramente e não resultaram em associações significativas. Uma restrição semelhante pode ser vista nas medidas de apego desenvolvido, tanto das mães quanto dos seus filhos, que não foram realizadas, devido a dificuldades de obtenção de informação sobre eventos passados, bem como aos modestos recursos da pesquisa.

Além disso, o design da pesquisa e o tamanho da amostra não permitem a eliminação de hipóteses rivais. A objeção apresentada por Wachs (2000) às relações encontradas é pertinente a este estudo: não se pode eliminar a possibilidade de que as diferenças no calendário maturacional sejam geneticamente determinadas e que a precocidade da vida sexual afete o relacionamento conjugal, este, por sua vez, gerando um ambiente familiar instável, com resultados negativos sobre o desenvolvimento da criança. Embora haja alguma evidência de que o efeito de antecipação da vida reprodutiva devido a fatores psicossociais operando nos primeiros anos de vida é independente dos efeitos genéticos (Graber et al., 1995), qualquer interpretação dos dados aqui apresentados deve se ater às possibilidades do design empregado, com as limitações pertinentes.

Além disso, uma vez que variáveis como renda e escolaridade dos pais estão fortemente associadas a resultados no desenvolvimento em muitas esferas, é claro que as famílias compostas pelos dois genitores têm, em geral, mais recursos materiais do que aquelas formadas por um dos genitores. Isso torna difícil separar dimensões materiais e psicológicas do ambiente familiar e seu efeito no desenvolvimento da criança.

Apesar dessas limitações, os resultados encontrados representam uma abordagem inicial à teoria do investimento parental nas condições contemporâneas brasileiras. Essa abordagem inicial sugere uma seqüência de relações entre eventos em diferentes momentos do ciclo vital, afetados pelas condições ecológicas vigentes.

No entanto, para avançar no entendimento e teste da teoria, é necessário investigar em detalhes cada segmento da cadeia de associações, controlando as variáveis que podem mediar as relações, bem como as geradoras de confusão. Assim, este estudo pode representar uma abertura para um programa de pesquisa de amplo alcance, a ser empreendido pela psicologia brasileira de orientação evolucionista.

\section{Agradecimentos}

Os autores agradecem o apoio do CNPq (Conselho Nacional de Desenvolvimento Científico e Tecnológico).

\section{Referências}

AVSI - Associação dos Voluntários para o Serviço Internacional (1999). Retrato da comunidade de Novos Alagados - segunda etapa. Salvador: Autor.

Barber, N. (2003). Paternal investment prospects and cross-national differences in single parenthood. Cross-cultural Research: The Journal of Comparative Social Sciences, 37(2), 163-177.
Bayley, N. (1993). Bayley scales of infant development ( $2^{\underline{a}}$ ed.). San Antonio: The Psychological Corporation.

Belsky, J., Steinberg, L., \& Draper, P. (1991). Childhood experience, interpersonal development, and reproductive strategy: an evolutionary theory of socialization. Child Development, 62(4), 682-685.

Eickmann, S. H., Lira, P. I. C., \& Lima, M. C. (2002). Desenvolvimento mental e motor aos 24 meses de crianças nascidas a termo com baixo peso. Arquivos de Neuro-Psiquiatria, 60, 748-754.

Ellis, B. J., Bates, J. E., Dodge, K. A., Fergusson, D. M., Harwwod, L. J., Pettit, G. S., \& Woodward, L. (2003). Does father absence place daughters at special risk for early sexual activity and teenage pregnancy? Child Development, 74, 801-821.

Ellis, B. J., \& Garber, J. (2000). Psychosocial antecedents of variation in girls' pubertal timing: maternal depression, stepfather presence, and marital and family stress. Child Development, 71, 485-501.

Gagliardo, H. G. R. G., Gonçalves, V. M. G., Lima, M. C. M. P., Francozo, M. F. C., \& Aranha Neto, A. (2004). Função visual e controle motor apendicular em lactentes pequenos para a idade gestacional. Arquivos de Neuro-Psiquiatria, 62, 300-306.

Graber, J. A., Brooks-Gunn, J., \& Warren, M. P. (1995). The antecedents of menarcheal age: heredity, family environment, and stressful life events. Child Development, 66, 346-359.

Hrdy, S. B. (2000). Mãe natureza: uma visão feminina da evolução, maternidade, filhos e seleção natural. São Paulo: Campus.

Keller, H. (1996). Evolutionary approaches. In J. W. Berry, Y. H. Poortinga \& J. Pandey (Orgs.), Handbook of Cross-cultural Psychology, vol. 1, Theory and Method (pp. 215-256). Boston: Allyn \& Bacon.

Keller, H. (1998a). Diferentes caminhos de socialização até a adolescência. Revista Brasileira de Crescimento e Desenvolvimento Humano, 8(1/2), 1-14.

Keller, H. (1998b). Personality formation in different cultures. A crosscultural study program for longitudinal assessments. Projeto de pesquisa não-publicado.

Lago, M. J. (2002). Idade da menarca, condições sócio-econômicas na infância e estatura na vida adulta: o estudo Pró-Saúde. Tese de doutorado, Instituto de Medicina Social, Universidade Federal do Rio de Janeiro. Resumo obtido de http://bases.bireme.br, em 04 de abril de 2005.

Mello, B. B. A., Gonçalves, V. M. G., \& Souza, E. A. P. (2004). Comportamento de lactentes nascidos a termo pequenos para a idade gestacional no primeiro trimestre da vida. Arquivos de Neuro-Psiquiatria, 62, 1046-1051.

Moffit, T. E., Caspi, A., Belsky, J., \& Silva, P. A. (1992). Childhood experience and the onset of menarche: a test of a sociobiological model. Child Development, 63(1), 47-58.

Moreira, L. V. C. (1999). Concepções de mães usuárias de creche sobre educação de filhos. Dissertação de mestrado não-publicada, Faculdade de Educação, Universidade Federal da Bahia, Salvador.

Moreira, L. V. C., \& Lordelo, E. R. (2002). Creche em ambiente urbano pobre: ressonâncias no ecossistema desenvolvimental. Interação em Psicologia, 6(1), 1-13.

Moss, E., Cyr, C., \& Dubois-Comtois, K. (2004). Attachment at early school age and developmental risk: examining family contexts and behavior problems of controlling-caregiving, controlling-punitive, and behaviorally disorganized children. Developmental Psychology, 49, 519-532.

Moss, E., \& St-Laurent, D. (2001). Attachment at school age and academic performance. Child Development, 37, 863-874.

Pettit, G. S., \& Bates, J. E. (1999). Quality of early family relationships and individual differences in the timing of pubertal maturation in girls: a longitudinal test of an evolutionary model. Journal of Personality and Social Psychology, 77(2), 387-401. 
Picanço, M. R. A. (1995). A idade da menarca da menina brasileira: os fatores sócio-econômicos e as diferenças regionais. Dissertação de mestrado nãopublicada, Instituto Fernandes Figueira, Rio de Janeiro. Resumo obtido de http://bases.bireme.br, em 04 de abril de 2005.

Quinlan, R. J., Quinlan, M. B., \& Flinn, M. V. (2003). Parental investment and age at weaning in a Caribbean village. Evolution and Human Behavior, 24, $1-16$.

Schor, N. (1990). Investigação sobre ocorrência de aborto em pacientes de hospital de centro urbano do Estado de São Paulo, Brasil. Revista de Saúde Pública, 24(2), 144-151.

Stams, G. J. J., Juffer, F., \& van-Ijzendoorn, M. H. (2002). Maternal sensitivity, infant attachment, and temperament in early childhood predict adjustment in middle childhood: the case of adopted children and their biologically unrelated parents. Developmental Psychology, 38, 806-821.
Tavares, C. H. F., Haeffner, L. S. B., Barbieri, M. A., Bettiol, H., Barbieri, M. R., \& Souza, L. (2000). Idade da menarca em escolares de uma comunidade rural do Sudeste do Brasil. Cadernos de Saúde Pública, 16, 709-715.

Wachs, T. D. (2000). Necessary but not sufficient: the respective roles of single and multiple influences on individual development. Washington: American Psychological Association.

Weschler, D. (1989). WIPPSI-R - Wechsler Preschool Scale of Intelligence Revised. San Antonio: The Psychological Corporation \& Harcout Brace Jovanovich.

Zerwes, E. P., \& Simões, P. M. (1993). Determinação da idade da menarca em relação ao estado nutricional, em escolares de estabelecimentos municipais, estaduais e particulares, da zona urbana da cidade de Pelotas, RS. Revista Brasileira de Ginecologia e Obstetrícia, 15(2), 67-68.

Eulina da Rocha Lordelo, doutora em Psicologia pela Universidade de São Paulo, é professora associada na Universidade Federal da Bahia e pesquisadora do CNPq. Endereço para correspondência: Rua Estrada de São Lázaro, 193 (Federação); Salvador, BA; CEP 40210-730. Tel.: (71) 3263-6352. Fax: (71) 3247-2800. E-mail: eulina@ufba.br

Carine Bastos da França é estudante de Psicologia na Universidade Federal da Bahia e bolsista de Iniciação Científica (CNPq/PIBIC/UFBa).

Lígia Maria dos Santos Lopes é psicóloga pela Universidade Federal da Bahia e bolsista de Iniciação Científica (CNPq/PIBIC/UFBa).

Maria del Pilar Ogando Dacal é psicóloga pela Universidade Federal da Bahia.

Cláudio Seal Carvalho é mestre em Psicologia pela Universidade Federal da Bahia e bolsista de Iniciação Científica (CNPq/PIBIC/UFBa).

Raquel Cardoso Guirra é psicóloga pela Universidade Federal da Bahia.

Anderson Almeida Chalhub é mestre em Psicologia pela Universidade Federal da Bahia e Bolsista de Apoio Técnico, CNPq. 